Algebraic 83 Geometric $\mathcal{T}$ opology

Volume 5 (2005) 1365-1388

Published: 14 October 2005

ATG

\title{
A categorification for the chromatic polynomial
}

\author{
Laure Helme-Guizon \\ YONGWU RONG
}

\begin{abstract}
For each graph we construct graded cohomology groups whose graded Euler characteristic is the chromatic polynomial of the graph. We show the cohomology groups satisfy a long exact sequence which corresponds to the well-known deletion-contraction rule. This work is motivated by Khovanov's work on categorification of the Jones polynomial of knots.
\end{abstract}

AMS Classification 57M27; 05C15

Keywords Khovanov homology, graph, chromatic polynomial

\section{Introduction}

In recent years, there have been a great deal of interests in Khovanov cohomology theory introduced in 3 . For each link $L$ in $S^{3}$, Khovanov defines a family of cohomology groups $H^{i j}(L)$ whose Euler characteristic $\sum_{i, j}(-1)^{i} q^{j} r k\left(H^{i, j}(L)\right)$ is the Jones polynomial of $L$. These groups were constructed through a categorification process which starts with a state sum of the Jones polynomial, constructs a group for each term in the summation, and then defines boundary maps between these groups appropriately. More recently, Khovanov and Rozansky have extended the theory for the HOMFLYPT polynomial [7] 8].

It is natural to ask if similar categorifications can be done for other invariants with state sums. In this paper, we establish a cohomology theory that categorifies the chromatic polynomial for graphs. This theory is based on the polynomial algebra with one variable $x$ satisfying $x^{2}=0$. We show our cohomology theory satisfies a long exact sequence which can be considered as a categorification for the well-known deletion-contraction rule for the chromatic polynomial. This exact sequence helps us to compute the cohomology groups of several classes of graphs. In particular, we point out that torsions do occur in the cohomology for some graphs.

In Section 2, we show our construction. This is explained in two equivalent settings: the cubic complex approach and the enhanced state approach. In 
Section 3, we discuss various properties of these cohomology groups, including the long exact sequence. Several computational examples are given. Some questions and more recent developments are discussed in Section 4.

Acknowledgements We wish to thank Joe Bonin for sharing his knowledge about graphs, and Mikhail Khovanov for his comments. The first author was partially supported by the CCAS Dean's Fellowship at the George Washington University. The second author was partially supported by NSF grant DMS0513918 .

\section{From a graph to cohomology groups}

\subsection{A diagram for the chromatic polynomial}

We begin with a brief review for the chromatic polynomial. Let $G$ be a graph with vertex set $V(G)$ and edge set $E(G)$. For each positive integer $\lambda$, let $\{1,2, \cdots, \lambda\}$ be the set of $\lambda$-colors. A $\lambda$-coloring of $G$ is an assignment of a $\lambda$-color to vertices of $G$ such that vertices that are connected by an edge in $G$ always have different colors. Let $P_{G}(\lambda)$ be the number of $\lambda$-colorings of $G$. It is well-known that $P_{G}(\lambda)$ satisfies the deletion-contraction relation

$$
P_{G}(\lambda)=P_{G-e}(\lambda)-P_{G / e}(\lambda)
$$

Furthermore, it is obvious that

$$
P_{N_{v}}(\lambda)=\lambda^{v} \text { where } N_{v} \text { is the graph with } v \text { vertices and no edges. }
$$

These two equations uniquely determines $P_{G}(\lambda)$. They also imply that $P_{G}(\lambda)$ is always a polynomial of $\lambda$, known as the chromatic polynomial.

There is another formula for $P_{G}(\lambda)$ that is useful for us. For each $s \subseteq E(G)$, let $[G: s]$ be the graph whose vertex set is $V(G)$ and whose edge set is $s$, let $k(s)$ be the number of connected components of $[G: s]$. We have

$$
P_{G}(\lambda)=\sum_{s \subseteq E(G)}(-1)^{|s|} \lambda^{k(s)} .
$$

Equivalently, grouping the terms $s$ with the same number of edges yields state sum formula

$$
P_{G}(\lambda)=\sum_{i \geq 0}(-1)^{i} \sum_{s \subseteq E(G),|s|=i} \lambda^{k(s)} .
$$


Formula (11) follows easily from the well-known state sum formula for the Tutte polynomial $T(G, x, y)=\sum_{s \subseteq E(G)}(x-1)^{r(E)-r(s)}(y-1)^{|s|-r(s)}$, where the rank function $r(s)$ is the number of vertices of $G$ minus the number of connected components of $[G: s]$. One simply applies the known relation $P(G, \lambda)=$ $(-1)^{r(E)} \lambda^{k(G)} T(G ; 1-\lambda, 0)$ between the two polynomials.

Our chain complex will depend on an ordering of the edges of the graph. Thus, let $G=(V, E)$ be a graph with an ordering on its $n$ edges. For each $s \subseteq E$, the spanning subgraph $[G: s]$ (a spanning subgraph of $G$ is one that contains all the vertices of $G$ ) can be described unambiguously by an element $\varepsilon=\left(\varepsilon_{1}, \varepsilon_{2}, \ldots ., \varepsilon_{n}\right)$ of $\{0,1\}^{n}$ with the convention $\varepsilon_{k}=1$ if the edge $e_{k}$ is in $s$ and $\varepsilon_{k}=0$ otherwise. This $\varepsilon$ is called the label of $s$ and will be denoted by $\varepsilon_{s}$ or simply $\varepsilon$. Conversely, to any $\varepsilon \in\{0,1\}^{n}$, we can associate a set $s_{\varepsilon}$ of edges of $G$ that corresponds to $\varepsilon$. When we think of $s$ in terms of the label $\varepsilon$, we may refer to the graph $[G: s]$ as $G_{\varepsilon}$.

The state sum formula in (2) can be illustrated in Figure 1. With the variable change $\lambda=1+q$, this gives the chromatic polynomial.

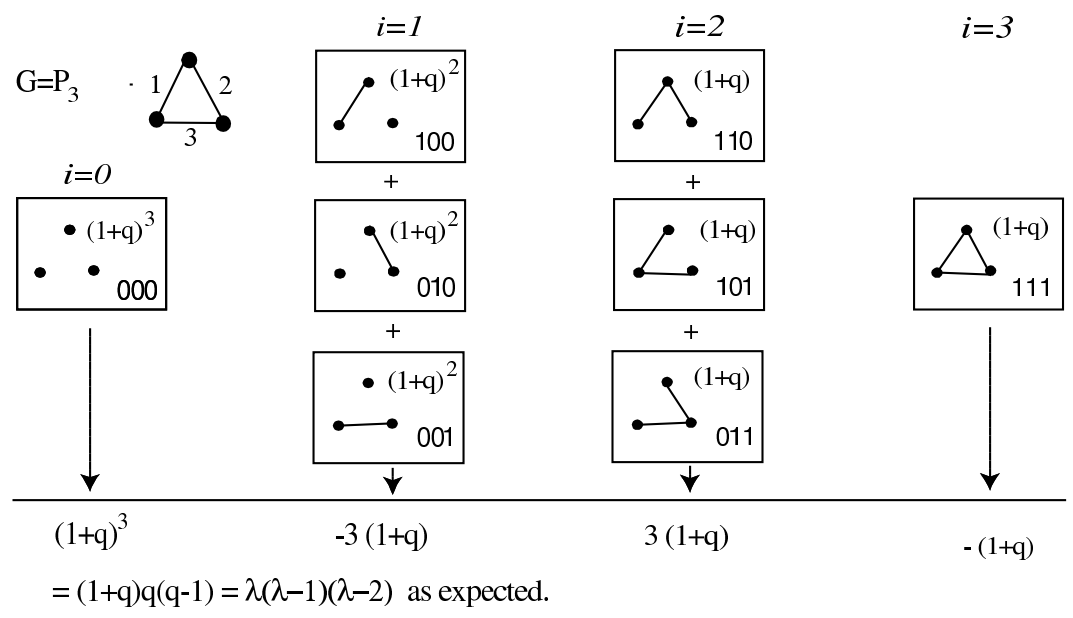

Figure 1: Diagram for a state sum computation of the Chromatic Polynomial when $G=P_{3}$

Each subset of edges $s$ (represented in Figure 1 by a labelled rectangle) corresponds to a term in the state sum and therefore will be called a state. Equivalently, if we think of a state in term of its label, we may call it a vertex of the cube $\{0,1\}^{n}$.

Each state corresponds to a subset $s$ of $E$, the n-list of $0^{\prime} s$ and $1^{\prime} s$ at the 
bottom of each rectangle is its label and the term of the form $(1+q)^{k(s)}=\lambda^{k(s)}$ is its contribution to the chromatic polynomial (without sign).

Note that we have drawn all the states that have the same number of edges in the same column, so that the column with label $i=i_{0}$ contains all the states with $i_{0}$ edges. Such states are called the states of height $i_{0}$. We denote the height of a state with label $\varepsilon$ by $|\varepsilon|$.

\subsection{The cubic complex construction of the chain complex}

\subsubsection{The chain groups}

We are going to assign a graded $\mathbb{Z}$-module to each state and define a notion of graded dimension so that the $(1+q)^{k}$ that appears in the rectangle is the graded dimension of this $\mathbb{Z}$-module. The construction is similar to Bar-Natan's description for the Khovanov cohomology for knots and links [1].

\section{Graded dimension of a graded $\mathbb{Z}$-module}

Definition 2.1 Let $\mathcal{M}=\oplus_{j} M_{j}$ be a graded $\mathbb{Z}$-module where $\left\{M_{j}\right\}$ denotes the set of homogeneous elements with degree $j$. The graded dimension of $\mathcal{M}$ is the power series

$$
q \operatorname{dim} \mathcal{M}:=\sum_{j} q^{j} \cdot \operatorname{rank}\left(M_{j}\right) .
$$

where $\operatorname{rank}\left(M_{j}\right)=\operatorname{dim}_{\mathbb{Q}}\left(M_{j} \otimes \mathbb{Q}\right)$.

Remark 2.2 $\mathcal{M}$ may have torsion but the graded dimension will not detect it.

The direct sum and tensor product can be defined in the graded category in an obvious way. The following proposition follows easily from definition and the proof is omitted.

Proposition 2.3 Let $\mathcal{M}$ and $\mathcal{N}$ be a graded $\mathbb{Z}$-modules. Then $\mathcal{M} \oplus \mathcal{N}$ and $\mathcal{M} \otimes \mathcal{N}$ are both graded $\mathbb{Z}$-modules with:

$$
\begin{aligned}
& q \operatorname{dim}(\mathcal{M} \oplus \mathcal{N})=q \operatorname{dim}(\mathcal{M})+q \operatorname{dim}(\mathcal{N}) \quad \text { and } \\
& q \operatorname{dim}(\mathcal{M} \otimes \mathcal{N})=q \operatorname{dim}(\mathcal{M}) \cdot q \operatorname{dim}(\mathcal{N})
\end{aligned}
$$


Example 2.4 Let $M$ be the graded free $\mathbb{Z}$-module with two basis elements 1 and $x$ whose degrees are 0 and 1 respectively. We have $M=\mathbb{Z} \oplus \mathbb{Z} x$ and $q \operatorname{dim} M=1+q$. This is the $\mathbb{Z}$-module we will use to construct our chain complex. We have $q \operatorname{dim}\left(M^{\otimes k}\right)=(1+q)^{k}$. In order to avoid confusion, we reserve the notation straight $M$ for this specific module and use $\mathcal{M}$ for a generic module.

Definition 2.5 Let $\{\ell\}$ be the "degree shift" operation on graded $\mathbb{Z}$-modules. That is, if $\mathcal{M}=\oplus_{j} M_{j}$ is a graded $\mathbb{Z}$-module where $M_{j}$ denotes the set of elements of $\mathcal{M}$ of degree $j$, we set $\mathcal{M}\{\ell\}_{j}:=M_{j-\ell}$ so that $q \operatorname{dim} \mathcal{M}\{\ell\}=$ $q^{\ell} . q \operatorname{dim} \mathcal{M}$. In other words, all the degrees are increased by $\ell$.

Therefore, $\mathbb{Z}\{1\}$ denotes $\mathbb{Z}$ with degree of each element being 1 . It is easy to check that $\mathcal{M} \otimes \mathbb{Z}\{1\} \cong \mathcal{M}\{1\}$, the $\mathbb{Z}$-module isomorphic to $\mathcal{M}$ with degree of every homogeneous element raised up by 1 .

We now explain our construction. Let $G$ be a graph with $n$ ordered edges, and let $M$ be as in Example 2.4. For each vertex $\varepsilon=\left(\varepsilon_{1}, \varepsilon_{2}, \ldots, \varepsilon_{n}\right)$ of the cube $\{0,1\}^{n}$, let $k_{\varepsilon}$ be the number of components of $G_{\varepsilon}$. We assign a copy of $M$ to each connected component and then take tensor product. This yields a graded free $\mathbb{Z}$-module $M_{\varepsilon}(G)=M^{\otimes k_{\varepsilon}}$. Now, $q \operatorname{dim} M_{\varepsilon}(G)$ is the polynomial that appears in the vertex $\varepsilon$ of the cube in Figure 1 .

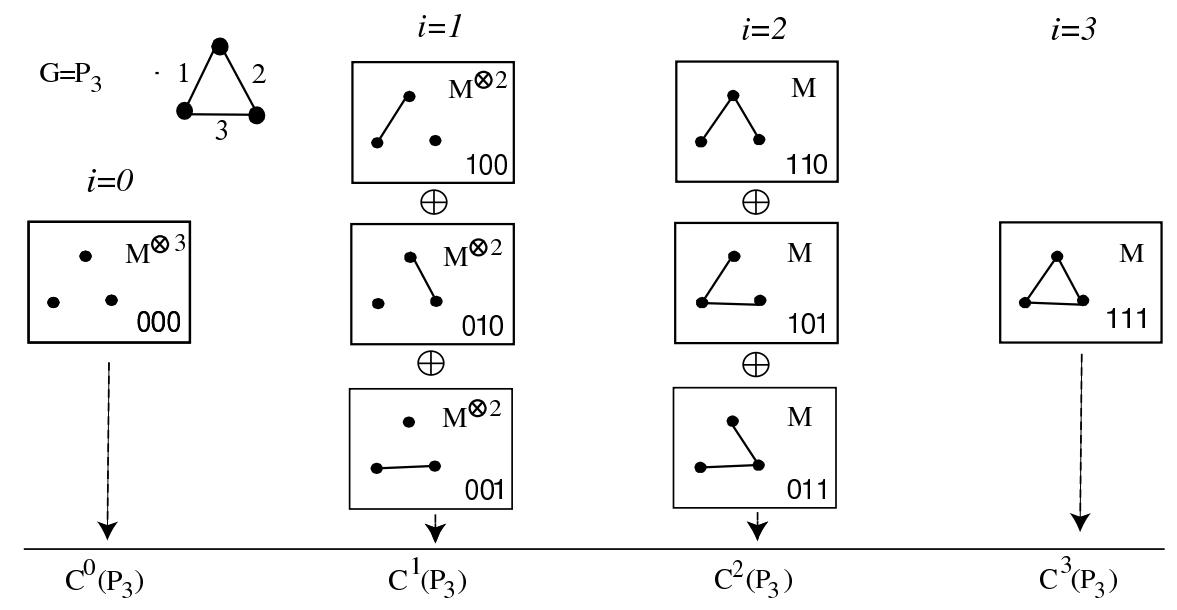

Figure 2: The chain groups $C^{i}(G)$

To get the chain groups, we "flatten" the cube by taking direct sums along the columns. A more precise definition is: 
Definition 2.6 We set the $i$-th chain group $C^{i}(G)$ of the chain complex $\mathcal{C}(G)$ to be the direct sum of all $\mathbb{Z}$-modules at height $i$, i.e. $C^{i}(G)=\underset{|\varepsilon|=i}{\oplus} M_{\varepsilon}(G)$.

The grading is given by the degree of the elements and we can write the $i$-th chain group as $C^{i}(G)=\oplus_{j \geq 0} C^{i, j}(G)$ where $C^{i, j}(G)$ denotes the elements of degree $j$ of $C^{i}(G)$.

For example, the elements of degree one of $C^{1}\left(P_{3}\right)$ are the linear combinations with coefficients in $\mathbb{Z}$ of the six elements shown in Figure 3 .

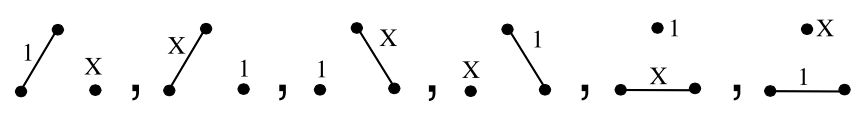

Figure 3: Basis elements of the free $\mathbb{Z}$-module $C^{1,1}\left(P_{3}\right)$

These elements form a basis of the free $\mathbb{Z}$-module $C^{1,1}\left(P_{3}\right)$. This will lead to a second description of our chain complex explained in section 2.3 .

\section{Graded chain complex, graded Euler characteristic}

Definition 2.7 Let $\mathcal{M}=\oplus_{j} M_{j}$ and $\mathcal{N}=\oplus_{j} N_{j}$ be graded $\mathbb{Z}$-modules where $M_{j}$ (resp. $N_{j}$ ) denotes the set of elements of $\mathcal{M}$ (resp. $\mathcal{N}$ ) of degree $j$.

A $\mathbb{Z}$-module map $\alpha: \mathcal{M} \rightarrow \mathcal{N}$ is said to be graded with degree $d$ if $\alpha\left(M_{j}\right) \subseteq$ $N_{j+d}$, i.e. elements of degree $j$ are mapped to elements of degree $j+d$.

A graded chain complex is a chain complex for which the chain groups are graded $\mathbb{Z}$-modules and the differentials are graded.

Definition 2.8 The graded Euler characteristic $\chi_{q}(\mathcal{C})$ of a graded chain complex $\mathcal{C}$ is the alternating sum of the graded dimensions of its cohomology groups, i.e. $\chi_{q}(C)=\sum_{0 \leq i \leq n}(-1)^{i} \cdot q \operatorname{dim}\left(H^{i}\right)$.

The following was observed in [1. For convenience of the reader, we include a proof here.

Proposition 2.9 If the differential is degree preserving and all chain groups are finite dimensional, the graded Euler characteristic is also equal to the alternating sum of the graded dimensions of its chain groups i.e.

$$
\chi_{q}(C)=\sum_{0 \leq i \leq n}(-1)^{i} \cdot q \operatorname{dim}\left(H^{i}\right)=\sum_{0 \leq i \leq n}(-1)^{i} \cdot q \operatorname{dim}\left(C^{i}\right) .
$$


Proof The corresponding result for the non-graded case is well known. That is, for a finite chain complex $\mathcal{C}=0 \rightarrow C^{0} \rightarrow C^{1} \rightarrow \ldots \rightarrow C^{n} \rightarrow 0$ with cohomology groups $H^{0}, H^{1}, \ldots, H^{n}$, if all the chain groups are finite dimensional then the Euler characteristic $\chi(\mathcal{C})=\sum_{0 \leq i \leq n}(-1)^{i} \operatorname{rank}\left(H^{i}\right)$ is also equal to $\sum_{0 \leq i \leq n}(-1)^{i} \operatorname{rank}\left(C^{i}\right)$.

Now, let $\mathcal{C}$ be a graded chain complex with a degree preserving differential. With the above notations, decomposing elements by degree yields $C^{i}=\underset{j \geq 0}{\oplus}$ $C^{i, j}(G)$. Since the differential is degree preserving, the restriction to elements of degree $j$, i.e. $0 \rightarrow C^{0, j} \rightarrow C^{1, j} \rightarrow \ldots \rightarrow C^{n, j} \rightarrow 0$ is a chain complex. The previous equation for non-graded case tells us $\sum_{0 \leq i \leq n}(-1)^{i} \operatorname{rank}\left(H^{i, j}\right) \quad=\sum_{0 \leq i \leq n}$ $(-1)^{i} \operatorname{rank}\left(C^{i, j}\right)$. Now, multiply this by $q^{j}$ and take the sum over all values of $j$ and the result follows.

\subsubsection{The differential}

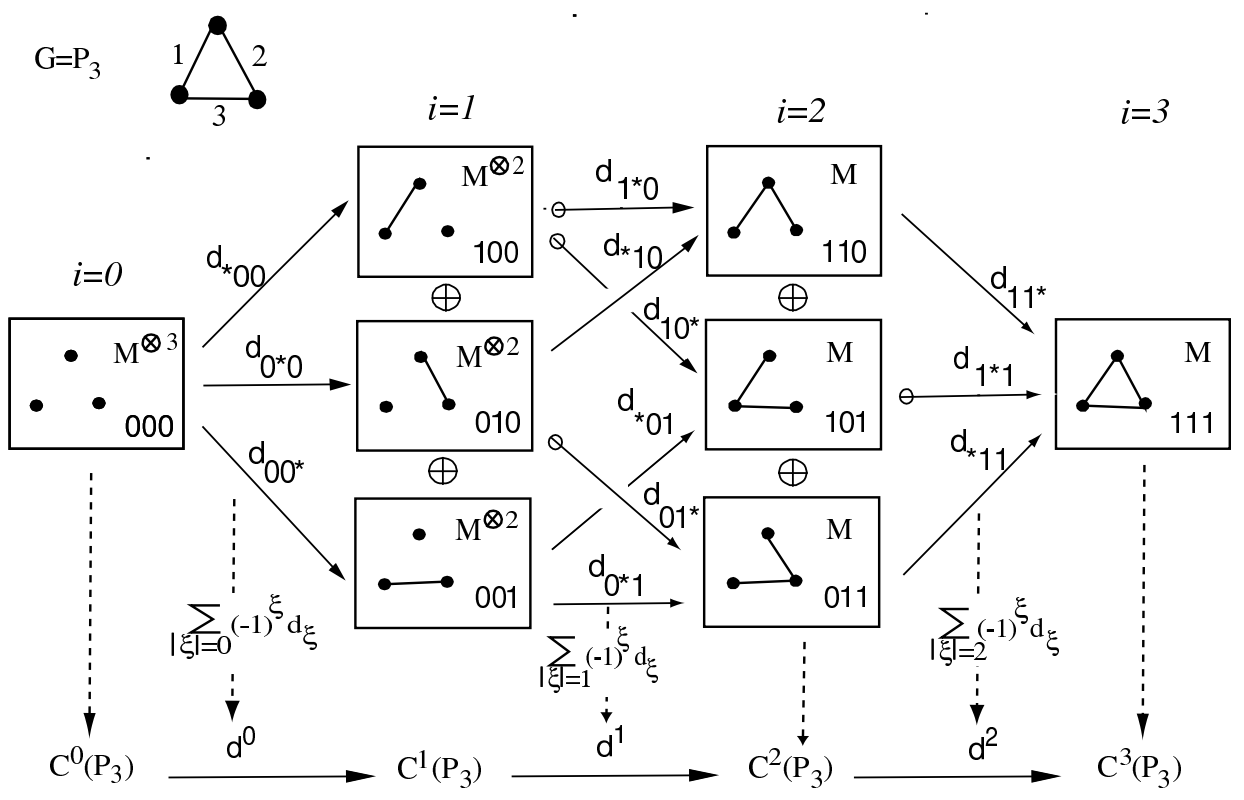

Figure 4: The differentials

Figure 4 shows what the maps look like. Details are as follows.

We will first turn the edges of the cube $\{0,1\}^{n}$ into maps between the $\mathbb{Z}$ modules corresponding to its ends. We call these maps per-edge maps. 
Recall that each vertex of the cube $\{0,1\}^{n}$ is labeled with some $\varepsilon=\left(\varepsilon_{1}, \ldots, \varepsilon_{n}\right) \in$ $\{0,1\}^{n}$.

\ For which pairs of vertices are there per-edge maps?

There is a map between the $\mathbb{Z}$-modules corresponding to two vertices if one of the markers $\varepsilon_{k}$ is changed from 0 to 1 when you go from the first vertex to the second vertex and all the other $\varepsilon_{k}$ are unchanged, and no map otherwise.

Denote by $\varepsilon$ the label of the first vertex. If the marker which is changed from 0 to 1 has index $k_{0}$ then the map will be labelled $d_{\varepsilon^{\prime}}^{+}$where $\varepsilon^{\prime}=\left(\varepsilon_{1}^{\prime}, \ldots, \varepsilon_{n}^{\prime}\right)$ with $\varepsilon_{k}^{\prime}=\varepsilon_{k}$ if $k \neq k_{0}$ and $\varepsilon_{k}^{\prime}=*$ if $k=k_{0}$.

For example, in the above diagram, the label $0 * 1$ of the map $d_{0 * 1}^{+}$means its domain is the $\mathbb{Z}$-module corresponding to the vertex labelled 001 and its target is the $\mathbb{Z}$-modules corresponding to the vertex labelled 011 . The superscript + indicates that we have not assigned signs to the map yet.

\ Definition of the per-edge maps

Changing exactly one marker from 0 to 1 corresponds to adding an edge.

$\triangle$ If adding that edge doesn't change the number of components, then the map is identity on $M^{\otimes k}$.

$\triangle$ If adding that edge decreases the number of components by one, then we set $d_{\varepsilon^{\prime}}^{+}: M^{\otimes k} \rightarrow M^{\otimes k-1}$ to be identity on the tensor factors corresponding to components that don't participate and to be the $\mathbb{Z}$-linear map $m: M \otimes M$ $\rightarrow M$ given by $m(1 \otimes 1)=1, m(1 \otimes x)=m(x \otimes 1)=x$ and $m(x \otimes x)=0$ on the two affected components.

Note that identity and $m$ are degree preserving so $d_{\varepsilon^{\prime}}^{+}$inherits this property.

\ "Flatten" to get the differential

The differential $d^{i}: C^{i}(G) \rightarrow C^{i+1}(G)$ of the chain complex $\mathcal{C}(G)$ is defined by $d^{i}:=\sum_{|\varepsilon|=i}(-1)^{\varepsilon} d_{\varepsilon}^{+}$where $|\varepsilon|$ is the number of $1^{\prime}$ 's in $\varepsilon$ and $(-1)^{\varepsilon}$ is defined in the next paragraph.

\ Assign a \pm 1 factor to each per-edge map $d_{\varepsilon}^{+}$

Equipped with the maps $d_{\varepsilon}^{+}$, the cube $\{0,1\}^{n}$ is commutative. This is because the multiplication map $m$ is associative and commutative. To get the differential $d$ to satisfy $d \circ d=0$, it is enough to assign a \pm 1 factor to these maps in the following way: Assign -1 to the maps that have an odd number of 1 's before the star in their label $\varepsilon$, and 1 to the others. This is what was denoted $(-1)^{\varepsilon}$ in the definition of the differential. 
In Figure 4 we have indicated the maps for which $(-1)^{\varepsilon}=-1$ by a little circle at the tail of the arrow.

A straightforward calculation implies:

Proposition 2.10 This defines a differential, that is, $d^{2}=0$.

Now, we really have a chain complex $\mathcal{C}(G)$ where the chain groups and the differential are defined as above. According to Proposition [2.9] we have:

Theorem 2.11 The Euler characteristic of this chain complex $\mathcal{C}(G)$ is equal to the chromatic polynomial of the graph $G$ evaluated at $\lambda=1+q$.

\subsubsection{Independence of ordering of edges}

Let $G$ be a graph with edges labeled 1 to $n$. For any permutation $\sigma$ of $\{1, . ., n\}$, we define $G_{\sigma}$ to be the same graph but with edges labeled as follows. The edge which was labeled $k$ in $G$ is labeled $\sigma(k)$ in $G_{\sigma}$. In other words, $G$ is obtained from $G_{\sigma}$ by permuting the labels of the edges of $G$ according to $\sigma$.

Theorem 2.12 The chain complexes $\mathcal{C}(G)$ and $\mathcal{C}\left(G_{\sigma}\right)$ are isomorphic and therefore, the cohomology groups are isomorphic. In other words, the cohomology groups are independent of the ordering of the edges so they are well defined graph invariants.

Proof. Since the group of permutations on $n$ elements is generated by the permutations of the form $(k, k+1)$ it is enough to prove the result when $\sigma=$ $(k, k+1)$.

We will define an isomorphism $f$ such that the following diagram commutes:

$$
\begin{array}{ccccc}
\mathcal{C}(G): & C^{0}(G) \stackrel{d^{0}}{\rightarrow} C^{1}(G) \stackrel{d^{1}}{\rightarrow} \ldots \stackrel{d^{i-1}}{\rightarrow} C^{i}(G) \stackrel{d^{i}}{\rightarrow} \ldots \stackrel{d^{n-1}}{\rightarrow} C^{n}(G) \\
\downarrow f f & \downarrow f & \downarrow f & \ldots & \downarrow
\end{array}
$$

It is enough to define $f$ restricted on each submodule $M_{\varepsilon}(G) \quad($ where $|\varepsilon|=i$ ) since $C^{i}(G)$ is the direct sum of these submodules.

For any subset $s$ of $E$ with $i$ edges, there is a state in $C^{i}(G)$ and one in $C^{i}\left(G_{\sigma}\right)$ that correspond exactly to those edges. Let $\varepsilon=\left(\varepsilon_{1}, \ldots, \varepsilon_{n}\right)$ stand for $\varepsilon_{s}(G)$, the label of $s$ in $G$. The situation is illustrated in Figure 5. 


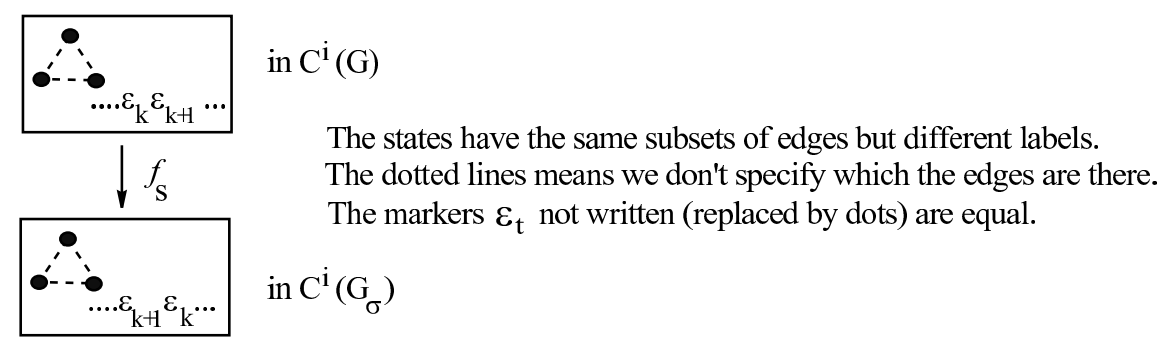

Figure 5: Impact of re-ordering of the edges

Let $f_{s}$ be the map between these two states that is equal to $-i d$ if $\varepsilon_{k}=\varepsilon_{k+1}=1$ and equal to $i d$ otherwise.

Let $f: C^{i}(G) \rightarrow C^{i}\left(G_{\sigma}\right)$ be defined by $f=\underset{|s|=i}{\bigoplus} f_{s}$.

$f$ is obviously an isomorphism. The fact that the diagram commutes can be checked by looking at the four cases $\left(\varepsilon_{k}, \varepsilon_{k+1}\right)=(0,0),\left(\varepsilon_{k}, \varepsilon_{k+1}\right)=(1,0)$, $\left(\varepsilon_{k}, \varepsilon_{k+1}\right)=(0,1)$ and $\left(\varepsilon_{k}, \varepsilon_{k+1}\right)=(1,1)$.

\subsubsection{A Poincaré polynomial}

We define a two-variable polynomial $R_{G}(t, q)$ by $R_{G}(t, q)=\sum_{0 \leq i \leq n} t^{i} \cdot q \operatorname{dim}\left(H^{i}(G)\right)$.

In the following proposition, (a) follows immediately from Theorem 2.12 and (b) follows from our construction.

Proposition 2.13 (a) The polynomial $R_{G}(t, q)$ depends only on the graph.

(b) The chromatic polynomial is a specialization of $R_{G}(t, q)$ at $t=-1$.

This polynomial is a convenient way to store the information about the free part of the cohomology groups and is, by construction, enough to recover the chromatic polynomial.

\subsection{Another description: The enhanced state construction}

These cohomology groups have another description that is similar to Viro's description for the Khovanov cohomology for knots [13. We explain the details below. 
Let $\{1, x\}$ be a set of colors, and $*$ be the product defined by

$$
1 * 1=1,1 * x=x * 1=x \text { and } x * x=0
$$

Let $G=(V, E)$ be a graph with an ordering on its edges. An enhanced state of $G$ is $S=(s, c)$, where $s \subseteq E$ and $c$ is an assignment of 1 or $x$ to each connected component of the spanning subgraph $[G: s]$. For each enhanced state $S$, define

$$
i(S)=\# \text { of edges in } s, \text { and } j(S)=\# \text { of } x \text { in } c .
$$

Note that $i(S)$ depends only on the underlying state $s$, not on the color assignment that makes it an enhanced state, so we may write it as $i(s)$.

Let $C^{i, j}(G):=\operatorname{Span}\langle S| S$ is an enhanced state of $G$ with $\left.i(S)=i, j(S)=j\right\rangle$, where the span is taken over $\mathbb{Z}$.

We define the differential

$$
d: C^{i . j}(G) \rightarrow C^{i+1, j}(G)
$$

as follows. For each enhanced state $S=(s, c)$ in $C^{i, j}(G)$, define $d(S) \in$ $C^{i+1, j}(G)$ by

$$
d(S)=\sum_{e \in E(G)-s}(-1)^{n(e)} S_{e}
$$

where $n(e)$ is the number of edges in $s$ that are ordered before $e, S_{e}$ is an enhanced state or 0 defined as follows. Let $s_{e}=s \cup\{e\}$. Let $E_{1}, \cdots, E_{k}$ be the components of $[G: s]$. If $e$ connects some $E_{i}$, say $E_{1}$, to itself, then the components of $[G:(s \cup\{e\})]$ are $E_{1} \cup\{e\}, E_{2}, \cdots, E_{k}$. We define $c_{e}\left(E_{1} \cup\right.$ $\{e\})=c\left(E_{1}\right), c_{e}\left(E_{2}\right)=c\left(E_{2}\right), \cdots, c_{e}\left(E_{k}\right)=c\left(E_{k}\right)$, and $S_{e}$ is the enhanced state $\left(s_{e}, c_{e}\right)$. If $e$ connects some $E_{i}$ to $E_{j}$, say $E_{1}$ to $E_{2}$, then the components of $\left[G: s_{e}\right]$ are $E_{1} \cup E_{2} \cup\{e\}, E_{3}, \cdots, E_{k}$. We define $c_{e}\left(E_{1} \cup E_{2} \cup\{e\}\right)=c\left(E_{1}\right) *$ $c\left(E_{2}\right), c_{e}\left(E_{3}\right)=c\left(E_{3}\right), \cdots, c_{e}\left(E_{k}\right)=c\left(E_{k}\right)$. Note that if $c\left(E_{1}\right)=c\left(E_{2}\right)=$ $x, c_{e}\left(E_{1} \cup E_{2} \cup\{e\}\right)=x * x=0$, and therefore $c_{e}$ is not considered as a coloring. In this case, we let $S_{e}=0$. In all other cases, $c_{e}$ is a coloring and we let $S_{e}$ be the enhanced state $\left(s_{e}, c_{e}\right)$.

One may find it helpful to think of $d$ as the operation that adds each edge not in $s$, adjusts the coloring using $*$, and then sums up the enhanced states using appropriate signs. In the case when an illegal color of 0 appears, due to the product $x * x=0$, the contribution from that edge is counted as 0 . 


\subsection{Equivalence of the two constructions}

At first sight, the two constructions look different because the cubic complex construction yields only one chain complex whereas the enhanced states construction gives rise to a sequence of chain complexes, one for each degree $j$. This can be easily solved by splitting the chain complex of the cubic complex construction into a sequence of chain complexes, one for each degree $j$. More precisely, let $\mathcal{C}=0 \rightarrow C^{0} \rightarrow C^{1} \rightarrow \ldots \rightarrow C^{n} \rightarrow 0$ be a graded chain complex with a degree preserving differential. Decomposing elements of each chain

group by degree yields $C^{i}=\underset{j \geq 0}{\oplus} C^{i, j}$. Since the differential is degree preserving, the restriction to elements of degree $j$, i.e. $0 \rightarrow C^{0, j} \rightarrow C^{1, j} \rightarrow \ldots \rightarrow C^{n, j} \rightarrow 0$ is a chain complex denoted by $\mathcal{C}^{j}$.

We are now ready to see that for a fixed $j$, the chain complexes obtained via the two construction are isomorphic. For this paragraph, denote the one obtained via the cubic complex construction by $\mathcal{C}^{j}$ and the one obtained via the enhanced state construction by $\widetilde{\mathcal{C}}^{j}$.

Both chain complexes have free chain groups so it is enough to define the chain map on basis elements. We will associate each enhanced state $S=(s, c)$ of $\widetilde{C}^{i, j}(G)$ to an unique basis element in $C^{i, j}(G)$ and show that this defines an isomorphism of chain complexes. First, $s \subseteq E(G)$ naturally corresponds to the vertex $\varepsilon=\left(\varepsilon_{1}, \cdots, \varepsilon_{n}\right)$ of the cube, where $\varepsilon_{k}=1$ if $e_{k} \in s$ and $\varepsilon_{k}=0$ otherwise. The corresponding $\mathbb{Z}$-module $M_{\varepsilon}(G)$ is obtained by assigning a copy of $M$ to each connected component of $[G, s]$ and then taking tensor product. The color $c$ naturally corresponds to the basis element $x_{1} \otimes \cdots \otimes x_{k}$ where $x_{\ell}$ is the color associated to the $\ell$-th component of $[G: s]$.

It is not difficult to see that this defines an isomorphism on the chain group that commutes with the differentials. Therefore, the two complexes are isomorphic.

\section{Properties}

In this section, we demonstrate some properties of our cohomology theory, as well as some computational examples.

\subsection{An exact sequence}

The chromatic polynomial satisfies a well-known deletion-contraction rule:

$$
P(G, \lambda)=P(G-e, \lambda)-P(G / e, \lambda) .
$$


Here we show that our cohomology groups satisfy a naturally constructed long exact sequence involving $G, G-e$, and $G / e$. Furthermore, by taking the Eulercharacteristic of the long exact sequence, we recover the deletion-contraction rule. Thus our long exact sequence can be considered as a categorification of the deletion-contraction rule.

We explain the exact sequence in terms of the enhanced state sum approach. Let $G$ be a graph and $e$ be an edge of $G$. We order the edges of $G$ so that $e$ is the last edge. This induces natural orderings on $G / e$ and on $G-e$ by deleting $e$ from the list. We define homomorphisms $\alpha_{i j}: C^{i-1, j}(G / e) \rightarrow C^{i, j}(G)$ and $\beta_{i j}: C^{i, j}(G) \rightarrow C^{i, j}(G-e)$. These two maps will be abbreviated by $\alpha$ and $\beta$ from now on. Let $v_{e}$ and $w_{e}$ be the two vertices in $G$ connected by $e$. Intuitively, $\alpha$ expands $v_{e}=w_{e}$ by adding $e$, and $\beta$ is the projection maps. We explain more details below.

First, given an enhanced state $S=(s, c)$ of $G / e$, let $\widetilde{s}=s \cup\{e\}$. The number of components of $[G / e: s]$ and $[G: \widetilde{s}]$ are the same. In fact, the components of $[G / e: s]$ and the components of $[G: \widetilde{s}]$ are the same except the one containing $v_{e}$ where $v_{e}$ in $G / e$ is replaced by $e$ in $G$. Thus, $c$ automatically yields a coloring of components of $[G: \widetilde{s}]$, which we denote by $\widetilde{c}$. Let $\alpha(S)=(\widetilde{s}, \widetilde{c})$. It is an enhanced state in $C^{i, j}(G)$. Extend $\alpha$ linearly and we obtain a homomorphism $\alpha: C^{i-1, j}(G / e) \rightarrow C^{i, j}(G)$.

Next, we define the map $\beta: C^{i, j}(G) \rightarrow C^{i, j}(G-e)$. Let $S=(s, c)$ be an enhanced state of $G$. If $e \notin s, S$ is automatically an enhanced state of $G-e$ and we define $\beta(S)=S$. If $e \in s$, we define $\beta(S)=0$. Again, we extend $\beta$ linearly to obtain the map $\beta: C^{i, j}(G) \rightarrow C^{i, j}(G-e)$.

One can sum up over $j$, and denote the maps by $\alpha_{i}: C^{i-1}(G / e) \rightarrow C^{i}(G)$ and $\beta_{i}: C^{i}(G) \rightarrow C^{i}(G-e)$. Again, they will be abbreviated by $\alpha$ and $\beta$. Both are degree preserving maps since the index $j$ is preserved.

Lemma 3.1 $\alpha$ and $\beta$ are chain maps such that $0 \rightarrow C^{i-1, j}(G / e) \stackrel{\alpha}{\rightarrow} C^{i, j}(G) \stackrel{\beta}{\rightarrow}$ $C^{i, j}(G-e) \rightarrow 0$ is a short exact sequence.

Proof First we show that $\alpha$ is a chain map. That is,

$$
\begin{gathered}
C^{i-1, j}(G / e) \stackrel{\alpha}{\rightarrow} C^{i, j}(G) \\
\downarrow d_{G / e} \quad \downarrow d_{G} \\
C^{i, j}(G / e) \stackrel{\alpha}{\rightarrow} C^{i+1, j}(G)
\end{gathered}
$$


commutes. Let $(s, c)$ be an enhanced state of $G / e$, we have

$$
d_{G} \circ \alpha((s, c))=d_{G}(s \cup\{e\}, \widetilde{c})=\sum_{e_{i} \in E(G)-(s \cup\{e\})}(-1)^{n_{G}\left(e_{i}\right)}\left(s \cup\left\{e, e_{i}\right\},(\widetilde{c})_{e_{i}}\right)
$$

where $n_{G}\left(e_{i}\right)$ is the number of edges in $s \cup\{e\}$ that are ordered before $e_{i}$ in $G$, and $(\widetilde{c})_{e_{i}}$ is the natural coloring inherited from $\widetilde{c}$ when adding the edge $e_{i}$ (see Section 2.3 for the description).

We also have $\alpha \circ d_{G / e}((s, c))=\alpha\left(\sum_{e_{i} \in E(G / e)-s}(-1)^{n_{G / e}\left(e_{i}\right)}\left(s \cup\left\{e_{i}\right\}, c_{e_{i}}\right)\right)=$ $\sum_{e_{i} \in E(G / e)-s}(-1)^{n_{G / e}\left(e_{i}\right)}\left(s \cup\left\{e_{i}, e\right\}, \widetilde{\left(c_{e_{i}}\right)}\right)$ where $n_{G / e}\left(e_{i}\right)$ is the number of edges in $s$ that are ordered before $e_{i}$ in $G / e$.

The two summations contain the same list of $e_{i}$ 's since $E(G)-(s \cup\{e\})=$ $E(G / e)-s$. It is also easy to see that $(\widetilde{c})_{e_{i}}=\widetilde{\left(c_{e_{i}}\right)}$. Finally, $n_{G}\left(e_{i}\right)=n_{G / e}\left(e_{i}\right)$ since $e$ is ordered last. It follows that $d_{G} \circ \alpha=\alpha \circ d_{G / e}$ and therefore $\alpha$ is a chain map.

Next, we show that $\beta$ is a chain map by proving the commutativity of

$$
\begin{gathered}
C^{i, j}(G) \stackrel{\beta}{\rightarrow} C^{i, j}(G-e) \\
\downarrow d_{G} \quad \downarrow d_{G-e} \\
C^{i+1, j}(G) \stackrel{\beta}{\rightarrow} C^{i+1, j}(G-e)
\end{gathered}
$$

Let $S=(s, c)$ be an enhanced state of $G$.

If $e \in s$, we have $\beta(S)=0$ and thus $d_{G-e} \circ \beta(S)=0$. We also have $d_{G}(S)=\sum_{e_{i} \in E(G)-s}(-1)^{n_{G}\left(e_{i}\right)}\left(s \cup\left\{e_{i}\right\}, c_{e_{i}}\right)$. Since $e \in s \cup\left\{e_{i}\right\}, \beta \circ d_{G}(S)=0$.

If $e \notin s$, we have $d_{G-e} \circ \beta(S)=d_{G-e}(S)=\sum_{e_{i} \in E(G-e)-s}(-1)^{n_{G-e}\left(e_{i}\right)}\left(s \cup\left\{e_{i}\right\}, c_{e_{i}}\right)$. We also have $d_{G}(S)=\sum_{e_{i} \in E(G)-s}(-1)^{n_{G}\left(e_{i}\right)}\left(s \cup\left\{e_{i}\right\}, c_{e_{i}}\right)=S_{1}+S_{2}$, where $S_{1}=\sum_{e_{i} \in E(G)-(s \cup\{e\})}(-1)^{n_{G}\left(e_{i}\right)}\left(s \cup\left\{e_{i}\right\}, c_{e_{i}}\right)$ corresponds to the terms with $e_{i} \neq$ $e$, and $S_{2}=(-1)^{n_{G}\left(e_{i}\right)}\left(s \cup\{e\}, c_{e}\right)$ corresponds to the term $e_{i}=e$. By our definition of $\beta, \beta\left(S_{1}\right)=S_{1}, \beta\left(S_{2}\right)=0$. Finally, $n_{G}\left(e_{i}\right)=n_{G-e}\left(e_{i}\right)$ since $e$ is ordered last, and it follows that $d_{G-e} \circ \beta(S)=\beta \circ d_{G}(S)$ as well in this case.

Finally, we prove the exactness. Each nonzero element in $C^{i-1, j}(G / e)$ can be written as $x=\sum n_{k}\left(s_{k}, c_{k}\right)$ where $n_{k} \neq 0$ and $\left(s_{k}, c_{k}\right)$ 's are distinct enhanced states of $G / e$. It is not hard to see that $\left(\tilde{s_{k}}, \tilde{c_{k}}\right)$ 's are distinct enhanced states of $G$. Thus $\alpha(x)=\sum n_{k}\left(\tilde{s_{k}}, \tilde{c_{k}}\right) \neq 0$ in $C^{i-1, j}(G)$. Hence ker $\alpha=0$. Next, 
$\operatorname{Im} \alpha=\operatorname{ker} \beta$, since both are $\operatorname{Span}\{(s, c) \mid(s, c)$ is an enhanced state of $G$ and $e \in s\}$. Last, $\beta$ is a projection map that maps onto $C^{i, j}(G-e)$.

The Zig-Zag lemma in homological algebra implies :

Theorem 3.2 Given a graph $G$ and an edge $e$ of $G$, for each $j$ there is a long exact sequence

$$
\begin{aligned}
& 0 \rightarrow H^{0, j}(G) \stackrel{\beta^{*}}{\rightarrow} H^{0, j}(G-e) \stackrel{\gamma^{*}}{\rightarrow} H^{0, j}(G / e) \stackrel{\alpha^{*}}{\rightarrow} H^{1, j}(G) \stackrel{\beta^{*}}{\rightarrow} H^{1, j}(G-e) \stackrel{\gamma^{*}}{\rightarrow} \\
& H^{1, j}(G / e) \rightarrow \ldots \rightarrow H^{i, j}(G) \stackrel{\beta^{*}}{\rightarrow} H^{i, j}(G-e) \stackrel{\gamma^{*}}{\rightarrow} H^{i, j}(G / e) \stackrel{\alpha^{*}}{\rightarrow} H^{i+1, j}(G) \rightarrow \ldots
\end{aligned}
$$

If we sum over $j$, we have a degree preserving long exact sequence:

$$
\begin{aligned}
& 0 \rightarrow H^{0}(G) \stackrel{\beta^{*}}{\rightarrow} H^{0}(G-e) \stackrel{\gamma^{*}}{\rightarrow} H^{0}(G / e) \stackrel{\alpha^{*}}{\rightarrow} H^{1}(G) \stackrel{\beta^{*}}{\rightarrow} H^{1}(G-e) \stackrel{\gamma^{*}}{\rightarrow} H^{1}(G / e) \rightarrow \\
& \ldots \rightarrow H^{i}(G) \stackrel{\beta^{*}}{\rightarrow} H^{i}(G-e) \stackrel{\gamma^{*}}{\rightarrow} H^{i}(G / e) \stackrel{\alpha^{*}}{\rightarrow} H^{i+1}(G) \rightarrow \ldots
\end{aligned}
$$

Remark 3.3 It is useful to understand how the maps $\alpha^{*}, \beta^{*}, \gamma^{*}$ act in an intuitive way. The descriptions for $\alpha$ and $\beta$ follows directly from our construction: $\alpha^{*}$ expands the edge $e, \beta^{*}$ is the projection map. The description for $\gamma^{*}$ follows from the standard diagram chasing argument in the zig-zag lemma and the result is as follows. For each cycle $z$ in $C^{i, j}(G-e)$ represented by the chain $\sum n_{k}\left(s_{k}, c_{k}\right), \gamma^{*}(z)$ is represented by the chain $(-1)^{i} \sum n_{k}\left(s_{k} \cup\{e\} / e,\left(c_{k}\right)_{e}\right)$, where $s_{k} \cup\{e\} / e$ is the subset of $E(G / e)$ obtained by adding $e$ to $s_{k}$ and then contracting $e$ to $v_{e},\left(c_{k}\right)_{e}$ is the coloring defined in Section 2.3. We leave it to the reader to verify the result.

\subsection{Graphs with loops or multiple edges}

We prove two propositions.

Proposition 3.4 If the graph has a loop then all the cohomology group are trivial.

Proof Let $G$ be a graph with a loop $\ell$. The exact sequence for $(G, \ell)$ is

$0 \rightarrow H^{0}(G) \rightarrow H^{0}(G-\ell) \stackrel{\gamma^{*}}{\rightarrow} H^{0}(G / \ell) \rightarrow H^{1}(G) \rightarrow H^{1}(G-\ell) \stackrel{\gamma^{*}}{\rightarrow} H^{1}(G / \ell) \rightarrow$ $\ldots \rightarrow H^{i}(G) \rightarrow H^{i}(G-\ell) \stackrel{\gamma^{*}}{\rightarrow} H^{i}(G / \ell) \rightarrow H^{i+1}(G) \rightarrow \ldots$

Using our description of the snake map $\gamma^{*}$ in Remark 3.3. we get that the map $H^{i}(G-\ell) \stackrel{\gamma^{*}}{\rightarrow} H^{i}(G / \ell)$ is $(-1)^{i} i d$. Therefore, $H^{i}(G)=0$ for all $i$. 
Proposition 3.5 The cohomology group are unchanged if you replace all the multiple edges of a graph by single edges.

Proof Assume that in some graph $G$ the edges $e_{1}$ and $e_{2}$ connect the same vertices. In $G / e_{2}, e_{1}$ becomes a loop so as observed earlier, $H^{i}\left(G / e_{2}\right)=0$ for all $i$. It follows from the long exact sequence that $H^{i}\left(G-e_{2}\right)$ and $H^{i}(G)$ are isomorphic groups. One can repeat the process until there is at most one edge connecting two given vertices without changing the cohomology groups.

\subsection{Disjoint union of two graphs}

Let $G_{1}$ and $G_{2}$ be two graphs and consider their disjoint union $G_{1} \sqcup G_{2}$. On the chain complex level, we have $\mathcal{C}\left(G_{1} \sqcup G_{2}\right)=\mathcal{C}\left(G_{1}\right) \otimes \mathcal{C}\left(G_{2}\right)$.

Theorem 3.6 For each $i \in \mathbb{N}$, we have :

$$
H^{i}\left(G_{1} \sqcup G_{2}\right) \cong\left[\underset{p+q=i}{\oplus} H^{p}\left(G_{1}\right) \otimes H^{q}\left(G_{2}\right)\right] \oplus\left[\underset{p+q=i+1}{\oplus} H^{p}\left(G_{1}\right) * H^{q}\left(G_{2}\right)\right]
$$

where ${ }^{*}$ denotes the torsion product of two abelian groups.

If we decompose the groups by degree, we have the following for all $i, j \in \mathbb{N}$ :

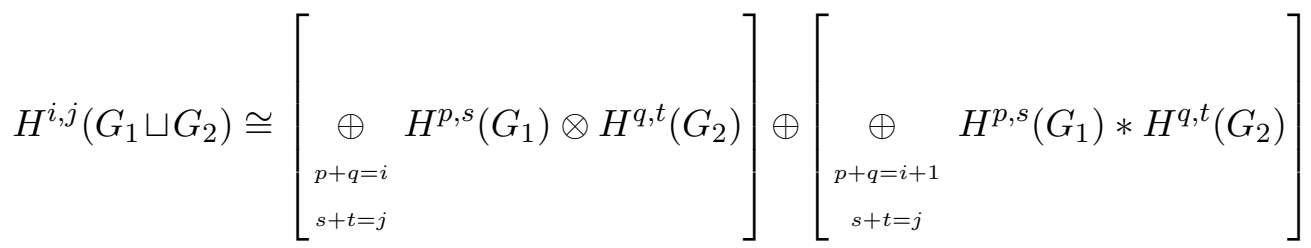

Proof This is a corollary of Künneth theorem, since the chains complexes $\mathcal{C}\left(G_{1}\right)$ and $\mathcal{C}\left(G_{2}\right)$ are free. See 10 for details about the Künneth theorem.

Corollary 3.7 The Poincaré polynomials are multiplicative under disjoint union i.e. $R_{G_{1} \sqcup G_{2}}(t, q)=R_{G_{1}}(t, q) \cdot R_{G_{2}}(t, q)$

Theorem 3.6 also implies

Example 3.8 Disjoint union with the one vertex graph:

$$
H^{i}(G \sqcup \bullet) \cong H^{i}(G) \otimes(\mathbb{Z} \oplus \mathbb{Z} x)
$$




\subsection{Adding or contracting a pendant edge}

An edge in a graph is called a pendant edge if the degree of one of its end points is one. Let $G$ be a graph and $e$ be a pendant edge of $G$. Let $G / e$ be the graph obtained by contracting $e$ to a point. We will study the relation between the cohomology groups of $G$ and $G / e$.

Recall that, for a given graded $\mathbb{Z}$-module $M, M\{1\}$ denotes the $\mathbb{Z}$-module isomorphic to $M$ with degree for each homogeneous element being shifted up by 1 . We have

Theorem 3.9 Let $e$ be a pendant edge in a graph $G$. For each $i, H^{i}(G) \cong$ $H^{i}(G / e)\{1\}$.

Proof Consider the operations of contracting and deleting $e$ in $G$. For convenience, denote the graph $G / e$ by $G_{1}$. We have $G / e=G_{1}$, and $G-e=G_{1} \sqcup\{v\}$, where $v$ is the end point of $e$ with $\operatorname{deg} v=1$. The exact sequence on $(G, e)$ is $0 \rightarrow H^{0}(G) \rightarrow H^{0}\left(G_{1} \sqcup\{v\}\right) \rightarrow H^{0}\left(G_{1}\right) \rightarrow \cdots \rightarrow H^{i}(G) \rightarrow H^{i}\left(G_{1} \sqcup\{v\}\right) \rightarrow$ $H^{i}\left(G_{1}\right) \rightarrow \cdots$

Thus we need to understand the map

$$
H^{i}\left(G_{1} \sqcup\{v\}\right) \stackrel{\gamma^{*}}{\rightarrow} H^{i}\left(G_{1}\right)
$$

By Theorem [3.6,

$$
H^{i}\left(G_{1} \sqcup\{v\}\right) \cong H^{i}\left(G_{1}\right) \otimes[\mathbb{Z} \oplus \mathbb{Z}\{1\}] \cong H^{i}\left(G_{1}\right) \oplus\left[H^{i}\left(G_{1}\right) \otimes \mathbb{Z}\{1\}\right]
$$

by a natural isomorphism $h_{*}$, which is induced by the isomorphism $h$ described as follows. Each enhanced state $S$ in $C^{i}\left(G_{1} \sqcup\{v\}\right)$ either assigns the color 1 to $v$, or the color $x$ to $v$. If it assigns 1 to $v, h$ sends $S$ to $(-1)^{|s|}\left(S_{1}, 0\right)$ where $S_{1}$ is the "restriction" of $S$ to $G_{1}$ and $|s|$ is the number of edges of the underlying set $s$ of $S=(s, c)$. If it assigns $x$ to $v, h$ sends $S$ to $(-1)^{|s|}\left(0, S_{1} \otimes x\right)$. This extends to a degree preserving isomorphism on chain groups and induces the isomorphism $h^{*}$ on cohomology groups.

We therefore will identify $H^{i}\left(G_{1} \sqcup\{v\}\right)$ with $H^{i}\left(G_{1}\right) \oplus\left[H^{i}\left(G_{1}\right) \otimes \mathbb{Z}\{1\}\right]$.

Claim $\gamma^{*} \circ\left(h^{*}\right)^{-1}: H^{i}\left(G_{1}\right) \oplus\left[H^{i}\left(G_{1}\right) \otimes \mathbb{Z}\{1\}\right] \rightarrow H^{i}\left(G_{1}\right)$ satisfies $\gamma^{*} \circ$ $\left(h^{*}\right)^{-1}(x, 0)=x$ for all $x \in H^{i}\left(G_{1}\right)$.

Proof of Claim Let $x$ be in $H^{i}\left(G_{1}\right) . \quad x$ is the equivalence class of a sum of terms of the form $(s, c)$ in $G_{1}$. Under the map $\left(h^{*}\right)^{-1}$, each of these terms 
is "extended" to be an element in $C^{i}\left(G_{1} \sqcup\{v\}\right)$ by adding $v$ to $\left[G_{1}: s\right]$ and assigning it the color 1 . The final result is then multiplied by $(-1)^{i}$.

The map $\gamma^{*}$ is described in Remark 3.3. Let $y$ be in $H^{i}\left(G_{1} \sqcup\{v\}\right) . y$ is the equivalence class of a sum of terms of the form $(s, c)$ in $H^{i}\left(G_{1} \sqcup\{v\}\right)$. Basically, for each each $(s, c), \gamma^{*}$ adds the edge $e$, adjusts the colorings, then contracts $e$ to a point and multiplies the result by $(-1)^{i}$. Hence applying $\left(h^{*}\right)^{-1}$ then $\gamma^{*}$ yields the original graph $G_{1}$. The color for each state in $x$ remains the same since $v$ is colored by 1 and multiplication by 1 is the identity map. This proves that $\gamma^{*} \circ\left(h^{*}\right)^{-1}(x, 0)=x$.

The claim implies that $\gamma^{*}$ is onto for each $i$. Thus the above long exact sequence becomes a collection of short exact sequences.

$$
0 \rightarrow H^{i}(G) \rightarrow H^{i}\left(G_{1} \sqcup\{v\}\right) \rightarrow H^{i}\left(G_{1}\right) \rightarrow 0
$$

After passing to the isomorphism $h_{*}$, the exact sequence becomes

$$
0 \rightarrow H^{i}(G) \rightarrow H^{i}\left(G_{1}\right) \oplus\left[H^{i}\left(G_{1}\right) \otimes \mathbb{Z}\{1\}\right] \rightarrow H^{i}\left(G_{1}\right) \rightarrow 0
$$

The next lemma implies that $H^{i}(G) \cong H^{i}(G) \otimes \mathbb{Z}\{1\}$.

Lemma 3.10 Let $A$ and $B$ be graded abelian groups, and $p: A \oplus B \rightarrow A$ be a degree preserving projection with $p(a, 0)=a$ for all $a \in A$. Then $\operatorname{ker} p \cong B$ via a degree preserving isomorphism.

Proof For each $b \in B$, let $a_{b}=p(0, b) \in A$. Then $p\left(-a_{b}, b\right)=0$ and therefore $\left(-a_{b}, b\right) \in \operatorname{ker} p$. Define $f(b)=\left(-a_{b}, b\right)$. It is a standard exercise to verify that $f$ is a degree preserving isomorphism from $B$ to $\operatorname{ker} p$.

\subsection{Trees, circuit graphs}

We describe the cohomology groups for several classes of graphs.

Example 3.11 Let $N_{1}$ be the graph with 1 vertex and no edge. Its chromatic polynomial is $P_{N_{1}}=\lambda=1+q$. The only enhanced states of $N_{1}$ are $(\emptyset, 1)$ and $(\emptyset, x)$, which generate $C^{0}\left(N_{1}\right)$. It follows that $H^{0}\left(N_{1}\right) \cong \mathbb{Z} \oplus \mathbb{Z}\{1\}$, and $H^{i}\left(N_{1}\right)=0$ for all $i \neq 0$.

Example 3.12 More generally, the graph with $v$ vertices and no edges is called the null graph of order $v$ and denoted by $N_{v}$. A similar argument implies

$$
H^{i}\left(N_{v}\right) \cong \begin{cases}(\mathbb{Z} \oplus \mathbb{Z}\{1\})^{\otimes v} & \text { if } i=0 \\ 0 & \text { if } i \neq 0\end{cases}
$$

This also follows from the Künneth type formula in Theorem (3.6). 
Example 3.13 Let $G=T_{n}$, a tree with $n$ edges. We can obtain $G$ by starting from a one point graph, and then adding pendant edges successively. Thus Theorem 3.9 and Example 3.11 imply

$$
H^{0}\left(T_{n}\right) \cong(\mathbb{Z} \oplus \mathbb{Z}\{1\})\{n\} \cong \mathbb{Z}\{n\} \oplus \mathbb{Z}\{n+1\}, H^{i}\left(T_{n}\right)=0 \text { for } i \neq 0 .
$$

A basis for $H^{0}\left(T_{n}\right)$ can be described as follows. Let $V\left(T_{n}\right)=\left\{v_{0}, v_{1}, \cdots, v_{n}\right\}$ be the set of vertices of $T_{n}$. Let $\sigma: V\left(T_{n}\right) \rightarrow\{ \pm 1\}$ be an assignment of \pm 1 to the vertices of $T_{n}$ such that vertices that are adjacent in $G$ always have opposite signs. It is easy to see that such a $\sigma$ exists (e.g. let $\sigma(v)=(-1)^{d\left(v_{0}, v\right)}$ where $d\left(v_{0}, v\right)$ is the number of edges in $T_{n}$ that connect $v_{0}$ to $\left.v\right)$. Furthermore, $\sigma$ is unique up to multiplication by -1 . For each $i=0,1, \cdots, n$, let $S_{i}=\left(\emptyset, c_{i}\right)$ be the enhanced state with $s=\emptyset, c_{i}$ assigns 1 to $v_{k}$ and $x$ to $v_{j}$ for each $j \neq i$. Let $\varepsilon_{1}=\sum_{i=0}^{n} \sigma\left(v_{k}\right) S_{i} \in C^{0}\left(T_{n}\right)$. Let $\varepsilon_{2}=(\emptyset, c)$ be the enhanced state with $s=\emptyset, c$ assigns $x$ to each vertex $v_{j}$ for $j=0, \cdots, n$. Then $\varepsilon_{1}$ is a generator for $\mathbb{Z}\{n\}$ and $\varepsilon_{2}$ is a generator for $\mathbb{Z}\{n+1\}$ in $H^{0}\left(T_{n}\right)$. An example is shown in Figure 6 below.

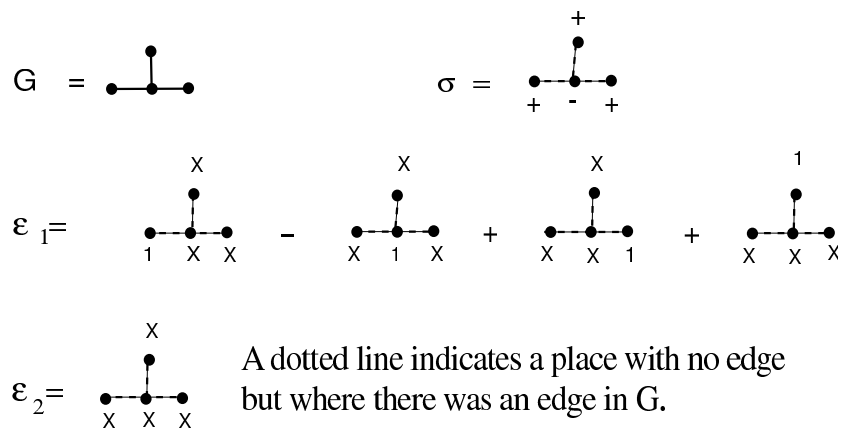

Figure 6: An example of basis for trees

Example 3.14 Circuit graph with n edges

Let $G=P_{n}$ be the polygon graph with $n$ edges, also known as the circuit graph or the cycle graph.

If $n=1, P_{1}$ is the graph with one vertex and one loop. By Proposition 3.4. $H^{i}\left(P_{1}\right)=0$ for each $i$.

If $n=2, P_{2}$ is the graph with two vertices connected by two parallel edges. By

Proposition 3.5 $H^{i}\left(P_{2}\right) \cong H^{i}\left(T_{1}\right) \cong \begin{cases}\mathbb{Z}\{1\} \oplus \mathbb{Z}\{2\} & \text { if } i=0 \\ 0 & \text { if } i \neq 0\end{cases}$ 
Next, let us assume $n>2$.

(In fact, the following method also holds for $n=2$ but the above method is a much easier way to get the result).

We label the vertices of $G=P_{n}$ by $v_{1}, \cdots, v_{n}$ monotonically so that each $v_{k}$ is adjacent to $v_{k+1}$ (here $\left.v_{n+1}=v_{1}\right)$. Let $e$ be the edge $v_{1} v_{n}$. Then $G-e$ is the tree with $n$ vertices $v_{1}, \cdots, v_{n}$ connected by a line segment running from $v_{1}$ to $v_{n}$, and $G / e$ is the polygon graph $P_{n-1}$ with vertices $v_{1}, \cdots, v_{n-1}$. The exact sequence on $(G, e)$ gives

$$
\cdots \rightarrow H^{i-1}(G) \rightarrow H^{i-1}(G-e) \rightarrow H^{i-1}(G / e) \rightarrow H^{i}(G) \rightarrow H^{i}(G-e) \rightarrow \cdots
$$

For $i \geq 2, H^{i-1}(G-e)=H^{i}(G-e)=0$ by Example 3.13. Thus $H^{i}(G) \cong$ $H^{i-1}(G / e)$, i.e. $H^{i}\left(P_{n}\right) \cong H^{i-1}\left(P_{n-1}\right)$ provided if $n \geq 2$ and $i \geq 2$. Applying this equation repeatedly, we have

$$
H^{i}\left(P_{n}\right) \cong \begin{cases}H^{1}\left(C_{n-i+1}\right) & \text { if } i \leq n \\ H^{i-n+1}\left(P_{1}\right)=0 & \text { if } i \geq n\end{cases}
$$

Thus it suffices to determine $H^{1}\left(P_{n}\right)$ and $H^{0}\left(P_{n}\right)$ for all $n$. Again, we examine part of the long exact sequence:

$$
0 \rightarrow H^{0}(G) \stackrel{\beta^{*}}{\rightarrow} H^{0}(G-e) \stackrel{\gamma^{*}}{\rightarrow} H^{0}(G / e) \stackrel{\alpha^{*}}{\rightarrow} H^{1}(G) \rightarrow 0
$$

Here, the last group $H^{1}(G-e)$ is 0 because $G-e$ is a tree. This exact sequence implies that

$$
H^{0}(G) \cong \operatorname{ker} \gamma^{*}, H^{1}(G) \cong H^{0}(G / e) / \operatorname{ker} \alpha^{*}=H^{0}(G / e) / \operatorname{Im} \gamma^{*}
$$

Thus we need to understand the map $\gamma^{*}: H^{0}(G-e) \rightarrow H^{0}(G / e)$.

This map can be described as follows. An $x$ in $H^{0}(G-e)$ is the equivalence class of a sum of terms of the form $(\emptyset, c)$ in $C^{0}(G-e)$. Each of these enhanced state $S=(\emptyset, c)$ is just a coloring of $v_{1}, \cdots, v_{n}$ by 1 or $x$. Under the map $\gamma^{*}$, $S=(\emptyset, c)$ is changed to $(\emptyset, \gamma(c))$ where $\gamma(c)$ is the coloring on $V(G / e)$ defined by $\gamma(c)\left(v_{k}\right)=c\left(v_{k}\right)$ for each $k \neq 1, n$, and $\gamma(c)\left(v_{1}\right)=c\left(v_{1}\right) * c\left(v_{n}\right)$. Basically, for each each $(s, c), \gamma^{*}$ adds the edge $e$, adjusts the colorings, then contracts $e$ to a point and multiplies the result by $(-1)^{i}$.

By Example (3.13), $H^{0}(G-e) \cong \mathbb{Z}\{n-1\} \oplus \mathbb{Z}\{n\}$ where $\mathbb{Z}\{n-1\}$ is generated by $\varepsilon_{1}$ and $\mathbb{Z}\{n\}$ is generated by $\varepsilon_{2}$. It is easy to see that $\gamma^{*}\left(\varepsilon_{2}\right)=0$ since all vertices are colored by $x$ in $\varepsilon_{2}$. As for $\gamma^{*}\left(\varepsilon_{1}\right)$, it will depend on the parity of $n$. We have $\varepsilon_{1}=S_{1}-S_{2}+\cdots+(-1)^{n-1} S_{n}$. For each $i \neq 1, n, \gamma^{*}\left(S_{i}\right)=0$ since both $v_{1}$ and $v_{n}$ are labeled by $x$ under $S_{i}$. For $i=1$ and $i=n$, $\gamma^{*}\left(S_{1}\right)=\gamma^{*}\left(S_{n}\right)=\varepsilon_{2}^{\prime}$ where $\varepsilon_{2}^{\prime}$ is the state of $C^{0}(G / e)$ that labels every vertex by $x$. Thus $\gamma^{*}\left(\varepsilon_{1}\right)=0$ if $n$ is even, and $\gamma\left(\varepsilon_{2}\right)=2 \varepsilon_{2}^{\prime}$ if $n$ is odd. 
It follows that ker $\gamma^{*}=\operatorname{Span}<\varepsilon_{1}, \varepsilon_{2}>$ if $n$ is even, and ker $\gamma^{*}=\operatorname{Span}<\varepsilon_{2}>$ if $n$ is odd. Therefore

$$
H^{0}\left(P_{n}\right) \cong \begin{cases}\mathbb{Z}\{n\} \oplus \mathbb{Z}\{n-1\} & \text { if } n \text { is even and } n \geq 2 \\ \mathbb{Z}\{n\} & \text { if } n \text { is odd and } n>2\end{cases}
$$

Next, we determine $H^{1}\left(P_{n}\right)$ using the same exact sequence. We follow the discussion above. If $n$ is even, $\gamma^{*}=0$, and therefore $H^{1}(G) \cong H^{0}(G / e) \cong$ $\mathbb{Z}\{n-1\}$. If $n$ is odd, $\operatorname{Im} \gamma^{*}=2 \mathbb{Z}\{n-1\}$ in $H^{0}(G / e)$. Therefore $H^{1}\left(P_{n}\right) \cong$ $H^{0}(G / e) / \operatorname{Im} \gamma^{*} \cong(\mathbb{Z}\{n-1\} \oplus \mathbb{Z}\{n-2\}) / 2 \mathbb{Z}\{n-1\} \cong \mathbb{Z}\{n-2\} \oplus \mathbb{Z}_{2}\{n-1\}$.

As a summary, we have

$$
\begin{gathered}
\text { For } i>0, H^{i}\left(P_{n}\right) \cong \begin{cases}\mathbb{Z}_{2}\{n-i\} \oplus \mathbb{Z}\{n-i-1\} & \text { if } n-i \geq 2 \text { and is even } \\
\mathbb{Z}\{n-i\} & \text { if } n-i \geq 2 \text { and is odd } \\
0 & \text { if } n-i \leq 1 .\end{cases} \\
\text { For } i=0, H^{0}\left(P_{n}\right) \cong \begin{cases}\mathbb{Z}\{n\} \oplus \mathbb{Z}\{n-1\} & \text { if } n \text { is even and } n \geq 2 \\
\mathbb{Z}\{n\} & \text { if } n \text { is odd and } n \geq 2 \\
0 & \text { if } n=0 .\end{cases}
\end{gathered}
$$

The following table illustrate our computational result (up to $n=6$ and $i=4$ ) for polygon graphs.

\begin{tabular}{|l|l|l|l|l|l|}
\hline$n \backslash i$ & $H^{0}$ & $H^{1}$ & $H^{2}$ & $H^{3}$ & $H^{4}$ \\
\hline$P_{1}$ & 0 & 0 & 0 & 0 & 0 \\
\hline$P_{2}$ & $\mathbb{Z}\{2\} \oplus \mathbb{Z}\{1\}$ & 0 & 0 & 0 & 0 \\
\hline$P_{3}$ & $\mathbb{Z}\{3\}$ & $\mathbb{Z}_{2}\{2\} \oplus \mathbb{Z}\{1\}$ & 0 & 0 & 0 \\
\hline$P_{4}$ & $\mathbb{Z}\{4\} \oplus \mathbb{Z}\{3\}$ & $\mathbb{Z}\{3\}$ & $\mathbb{Z}_{2}\{2\} \oplus \mathbb{Z}\{1\}$ & 0 & 0 \\
\hline$P_{5}$ & $\mathbb{Z}\{5\}$ & $\mathbb{Z}_{2}\{4\} \oplus \mathbb{Z}\{3\}$ & $\mathbb{Z}\{3\}$ & $\mathbb{Z}_{2}\{2\} \oplus \mathbb{Z}\{1\}$ & 0 \\
\hline$P_{6}$ & $\mathbb{Z}\{6\} \oplus \mathbb{Z}\{5\}$ & $\mathbb{Z}\{5\}$ & $\mathbb{Z}_{2}\{4\} \oplus \mathbb{Z}\{3\}$ & $\mathbb{Z}\{3\}$ & $\mathbb{Z}_{2}\{2\} \oplus \mathbb{Z}\{1\}$ \\
\hline
\end{tabular}

We note that, for all $n \geq 3, H^{*}\left(P_{n}\right)$ contains torsion. We will analyze such phenomenon for a general graph in future work.

\section{Further developments and future problems}

After this paper was posted on the arxiv in December 2004, there have been some new developments. Let us mention some of these below. 
First, our construction, which is based on the algebra $A_{2}:=\mathbb{Z}[x] /\left(x^{2}\right)$, can be generalized to any commutative graded algebras with a finite dimension at each degree. Such a generalization has been studied in [4].

Some computer computations of these cohomology groups have been done by Michael Chmutov. These computations lead to the following observation, which has now been proved by M. Chmutov, S. Chmutov, and Rong [2]. Namely, if we work on the algebra $A_{2}:=\mathbb{R}[x] /\left(x^{2}\right)$ over a field $\mathbb{R}$ of characteristic 0 , then $H^{i, n-i}(G) \cong H^{i+1, n-i-2}(G)$ for all $i$ and all $G$ expect for $i=0$ and $G$ is bipartite ("knight move" isomorphism). An analogous result for Khovanov's cohomology for alternating links/knots was proved by Lee [9]. While this implies that the free part of the cohomology groups is equivalent to the chromatic polynomial, it does not say anything about torsion. Moreover, we think this is due to fact that algebra used, $A_{2}$, is too simple. For more general algebras, we do not expect such equivalence result. Furthemore, some of the "twisted homologies" defined in [4] are stronger than the chromatic polynomial.

Let us add that the isomorphism classes of chain complexes are well defined graph invariants. They are certainly stronger than the cohomology groups since they can distinguish some graphs with loops.

The torsion of these homologies remains mysterious. Some studies have been carried out on torsion in [5], in joint work with J. Przytycki. In particular, we determine precisely, when the algebra is $A_{2}$, those graphs whose cohomology contains torsion. It remains to see where tosions occur and what torsion can occur.

Finally, we ask several questions that arise naturally in our work.

Problem 1 Understand geometric meanings of these cohomology groups The chromatic polynomial has a clear geometric interpretation. It is not clear what our cohomology groups measure. We note that these cohomology groups are not invariants of matroid type of the graphs. For example, the graph made of two triangles glued at one vertex and the graph which is the disjoint union of two triangles have the same matroid type but different chromatic polynomials, and therefore different cohomology groups.

Problem 2 Investigate functorial properties of the cohomology groups The classical homology theory is a functor: continuous maps between spaces induce homomorphism between homology groups. Khovanov's link cohomology $K h$ also satisfies a functorial property: a cobordism $C$ between two links $L_{1}$ and $L_{2}$ induces a homomorphism between the Khovanov homologies of $L_{1}$ and 
$L_{2}$. For our graph cohomology, we can associate homomorphisms between cohomology groups to each inclusion map of graphs. Essentially, it is the iteration of the map $\beta^{*}$ in the long exact sequence. We are not sure if this could lead to obstructions of one graph being a subgraph of another. In particular, can this be used to detect $K_{5}$ and $K_{3,3}$ in a given graph, and therefore determine the planarity of the graph? In any case, further study of this functor is worthwhile.

Problem 3 Carry out further study of torsions

First, what orders of torsion can we have? This will reflect the algebraic properties of the underlying algebra. As algebra varies, we can get torsions of arbitrary orders 4] 5]. For the algebra $A_{2}$, the only torsions based on examples we know are of order two. We would like to know whether other orders can occur. We would also like to know whether the distributions of torsion have any pattern, e.g. some kind of modified knight moves.

Problem 4 Study connections with Khovanov's link cohomology and other homologies

It is natural to ask for connections with link homologies given the connections between graph polynomials and link polynomials. A direct relation between $H^{i}(G)$ and the framed link cohomology (which corresponds to the Kauffman bracket) was given in [5], for all $i$ up to the length of the shortest cycle in $G$. Also, J. Przytycki has pointed out that our work in [HPR05] also shows connections of graph cohomology with other homology theories such as Hochschild homology of algebras.

Problem 5 Establish cohomology theories for various other graph polynomials, and for signed graphs

This is a natural question. Some work has already been done in this direction following our current paper. See, e.g. [1] 12] [6].

\section{References}

[1] D Bar-Natan, On Khovanov's categorification of the Jones polynomial, Algebr. Geom. Topol. 2 (2002) 337-370 MathReview

[2] M Chmutov, S Chmutov, Y Rong, Knight move for chromatic graph cohomology, in preparation

[3] M Khovanov, A categorification of the Jones polynomial, Duke Math. J. 101 (2000) 359-426 MathReview 
[4] L Helme-Guizon, Y Rong, Graph homologies from arbitrary algebras, arXiv:math.QA/0506023

[5] L Helme-Guizon, J Przytycki, Y Rong, Torsion in graph homologies, arXiv:math.GT/0507245

[6] F Jasso-Hernandez, Y Rong, A categorification for the Tutte polynomial, in preparation

[7] M Khovanov, L Rozansky, Matrix factorizations and link homology, arXiv:math.QA/0401268

[8] M Khovanov, L Rozansky, Matrix factorizations and link homology II, arXiv:math.QA/0505056

[9] E Lee, The support of the Khovanov's invariants for alternating knots, arXiv:math.GT/0201105

[10] J R Munkres, Elements of algebraic topology, Addison-Wesley Publishing Company, Menlo Park, CA (1984) MathReview

[11] M Stosic, Categorification of the dichromatic polynomial for graphs, arXiv:math.GT/0504239

[12] M Stosic, New categorifications of the chromatic and the dichromatic polynomials for graphs, arXiv:math.QA/0507290

[13] O Viro, Remarks on the definition of Khovanov homology, arXiv:math.GT/0202199

Department of Mathematics, The George Washington University

Washington, DC 20052, USA

Email: 1helmeg@yahoo.com, rong@gwu.edu

Received: 16 December 2004

Algebraic 83 Geometric Topology, Volume 5 (2005) 